\title{
Safe Charging for Wireless Power Transfer
}

\author{
Haipeng Dai*, Yunhuai Liu ${ }^{\dagger}$, Guihai Chen*‡, Xiaobing Wu*, Tian He ${ }^{\natural}$ \\ *State Key Laboratory for Novel Software Technology, Nanjing University, Nanjing, Jiangsu 210023, CHINA \\ $\dagger$ Third Research Institute of Ministry of Public Security, Shanghai, CHINA \\ $\ddagger$ Department of Computer Science and Engineering, Shanghai Jiao Tong University, Shanghai 200240, CHINA \\ $\S$ Computer Science and Engineering, University of Minnesota, Minneapolis, MN 55455, USA \\ Emails: \{dhpphd2003, yunhuai.liu\}@gmail.com, \{gchen, wuxb\}@nju.edu.cn, tianhe@cs.umn.edu
}

\begin{abstract}
As battery-powered mobile devices become more popular and energy hungry, wireless power transfer technology receives intensive interests, as it allows the power to be transferred from a charger to ambient devices wirelessly. The existing studies mainly focus on the power transfer efficiency but overlook the health impairments caused by RF exposure. In this paper, we study the Safe Charging Problem (SCP) of scheduling power chargers so that more energy can be received while no location in the field has electromagnetic radiation (EMR) exceeding a given threshold $R_{t}$. We prove that SCP is NP-hard and propose a solution which provably outperforms the optimal solution to SCP with a relaxed EMR threshold $(1-\epsilon) R_{t}$. Testbed results based on 8 Powercast TX91501 chargers validate our results. Extensive simulation results show that the gap between our solution and the optimal one is only $6.7 \%$ when $\epsilon=0.1$, while a naive greedy algorithm is $34.6 \%$ below our solution.
\end{abstract}

\section{INTRODUCTION}

As battery-powered mobile and portable devices become more popular and energy-hungry, energy conservation and scavenging schemes are increasingly important for the device usability. Among all such schemes, wireless power transfer technology [1] attracts intensive research and industry interest due to its convenience in applications. With this technology, energy can be transferred wirelessly from an energy storage to consuming devices such as RFIDs [2], sensors [3], cell phones [4], laptops [5], vehicles [6] and unmanned planes [7]. According to a recent report, the wireless power transfer market is expected to grow to US $\$ 23.7$ billion in 2015 [8].

In existing studies, researchers focused more on the energy charging efficiency and ubiquitousness, targeting at the minimal number of active chargers with more charging coverage (e.g., [9]-[20]). In practice, however, this is far from enough. Besides the charging efficiency, a more critical issue is the safety of the electromagnetic radiation (EMR). Though no study results have generally provided clear evidence of a relationship between EMR and the health impairments, there have been some statistically significant findings in certain positive cases. Exposure to high EMR [21] has been widely identified as a threat to human health. Its potential risks include but not limited to tissue impairment [22], brain tumor [23] and mental diseases [24]. Sec. II will give a more comprehensive survey on EMR safety issues and related regulations in the worldwide. A validated wireless charging scheme must comply with these regulations and guarantee the EMR safety in the field. No location should have the EMR value exceeding certain safety threshold.

With this practical and critical concern, in this paper, we propose and study the safe charging problem, attempting to strike the best tradeoff between the charging efficiency and EMR safety. Given a set of wireless power chargers and rechargeable devices, we are seeking the charger scheduling scheme so that the devices can obtain more power, while no location exceeds the EMR safety threshold.

Safe charging is a quite challenging problem which in general is NP-hard. The challenges are mainly due to the fact that the EMR constraints are imposed on every point in the field, which inevitably results in an infinite number of constraints. In addition, as we will show in later sections, the objective function is a non-convex one, which prevents the classical optimization method from applying directly. To overcome these challenges, we design constraint conversion and reduction techniques and apply approximation approaches, which enable us to transfer the problem to a traditional multidimensional 0/1 knapsack problem [25] and a FermatWeber problem [26], i.e., to find the optimal activation set of chargers maximizing the overall charging power under a limited number of constraints, and to find the point with the maximum EMR for a given active charger set. The constraints of the first problem are actually derived based on the outputs of the second problem.

The main contributions of this paper are as follows:

- To the best of our knowledge, this is the first work that considers charging efficiency under EMR safety concern. We formulate the problem as Safe Charging Problem (SCP), and prove that it is NP-hard.

- To deal with the problem, we design a series of novel techniques to transfer the problem to two traditional problems, namely a multidimensional 0/1 knapsack problem and a Fermat-Weber problem. The techniques include constraint conversion and reduction, bounded EMR function approximation, area discretization and expansion, and a tailored Fermat-Weber algorithm.

- We prove that for any given small number $\epsilon$, our solution outperforms the optimal solution to SCP with a relaxed EMR threshold $(1-\epsilon) R_{t}$, where $R_{t}$ is the original EMR threshold.

- To evaluate the performance of our algorithm, we build a testbed composed of 8 Powercast TX91501 chargers. Experimental results show that the maximal EMR in the 
field is $116.7 \mu \mathrm{W} / \mathrm{cm}^{2}$, which is just below the safety threshold of $125 \mu \mathrm{W} / \mathrm{cm}^{2}$. We also conduct comprehensive simulations and the gap between our solution and the optimal one is only $6.7 \%$ when $\epsilon=0.1$, while a naive greedy algorithm is $34.6 \%$ below our solution.

The remainder of the paper is organized as follows. In Sec. II, we elaborate on the motivation of this work. In Sec. III, we give preliminaries and a formal definition of the SCP problem. We introduce a near optimal solution to SCP in Sec. IV based on the theoretical results of MEP computation in the next section. Then, we present an approximation algorithm to MEP computation in Sec. V. Sec. VI and Sec. VII present extensive simulations and testbed experiments to verify our theoretical results. Sec. VIII reviews related work and Sec. IX concludes.

\section{Motivation}

In this section, we give a review of the risk of RF exposures and present some related regulations.

For the risk of RF exposures, there has been a significant body of research that focuses on the biological effects of RF signals on human body. For example, Olteanu et al. [22] investigated the harmful effect of metallic implant heating resulted from EMR around, which may lead to impairment of tissues. And it is reported that heating of tissue that exceeds 1 degree centigrade may interfere with behavioral and biological functions [27]. Gandhi et al. [28] found that children's heads absorb over two times of RF than adults, and absorption of the skull's bone marrow can be ten times greater than adults. Tissues of the fetus, such as the central nervous system, seem especially vulnerable to temperature rises caused by high EMR in various time windows, particularly during organogenesis [29]. Changes on gene/protein expression by RF exposure are also investigated. Leszczynskis group performed a pilot study on volunteers and showed that mobile phone radiation might alter protein expression in human skin cells [30]. Nittby et al. [31] found that a large number of genes were altered at hippocampus and cortex using four exposed and four control animals. Though no actual experiments have been conducted concerning the potential harm to people, a plenty of clinical studies such as [23] showed an increased risk of high EMR exposure for brain tumors. Besides, the link between RF exposure and mental diseases has also been confirmed. Experiments done on mice [24] showed that EMR causes transient and cumulative impairments in spatial and non-spatial memory.

Concerns about adverse consequences of EMR exposure have resulted in the establishment of exposure limits. These limits are codified in Title 47 of the Code of Federal Regulations (CFR) in the United States and Hygienic Standard for Environmental Electromagnetic Waves GB9175-88 [32] in China, and also contained in standards published by the International Commission on Non-Ionizing Radiation Protection (ICNIRP) [21] in most of Europe. For instance, the maximum allowed power density for frequency $915 \mathrm{M} \mathrm{Hz}$, the commonly used frequency band for wireless power transfer, by CFR, GB9175-88 and ICNIRP are $610 \mu \mathrm{W} / \mathrm{cm}^{2}, 40 \mu \mathrm{W} / \mathrm{cm}^{2}$ and $457.5 \mu \mathrm{W} / \mathrm{cm}^{2}$, respectively.

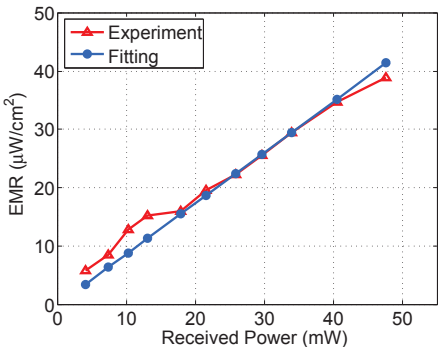

Fig. 1. EMR vs. Received Power

\section{Problem Statement}

Given the above concerns of the impairments of EMR, a desired charging scheme should strike a balance between the EMR limit and charging efficiency. In this paper, we assume all the chargers and rechargeable devices are static and deployed in fixed, known locations. In addition, we consider a simplified charger scheduling model in which chargers can be only in either of the on/off states. More complicated deployment schemes (e.g., mobile chargers) and scheduling models (e.g., adjustable charger power) are left for future work.

Ideally, the charging scheme should on one hand maximize the charging efficiency so that more charging energy can be harvested by the rechargeable devices, and on the other hand ensure the safety of the field by limiting the intensity of EMR at every position in the field. To achieve this goal, we will formulate the problem and propose our solutions in the remainder of this paper. In this section, we will first give the system and charging model, and then provide the formal problem statement. In the last of this section, we will present the main challenges we face when addressing the problem.

\section{A. System and charging model}

Assume there is a set of $n$ identical stationary wireless power chargers $S=\left\{s_{1}, s_{2}, \ldots, s_{n}\right\}$ deployed on a twodimensional plane, and we have a set of $m$ rechargeable devices $O=\left\{o_{1}, o_{2}, \ldots, o_{m}\right\}$ in the field too. The devices are capable of harvesting the wireless power originated from chargers to maintain their normal working.

In general, both the wireless charging power and the EMR intensity are related to the energy field strength. The received power $P_{r}(d)$ by a device can be quantified by an empirical model [13], i.e.,

$$
P_{r}(d)= \begin{cases}\frac{\alpha}{(d+\beta)^{2}}, & d \leq D \\ 0, & d>D\end{cases}
$$

where $d$ is the distance from the charger to the receiver, and $\alpha$ and $\beta$ are known constants determined by hardware of the charger and the receiver and the environment. Because of the hardware constraint, the energy field far away from the charger will be too small to be received by a node, and we use $D$ to denote the farthest distance a charger can reach.

For the charging, we assume the wireless power from multiple chargers to a receiver is additive [13], and define the charging utility to be proportional to the charging power, 
TABLE I

NOTATIONS

\begin{tabular}{r|l}
\hline Symbol & Meaning \\
\hline \hline$s_{i}, S$ & Charger $i$, charger set \\
$o_{j}, O$ & Device $j$, device set \\
$P_{r}(d)$ & Received power from distance $d$ \\
$D$ & Farthest distance a charger can reach \\
$d\left(s_{i}, o_{j}\right)$ & Distance from charger $s_{i}$ to device $o_{j}$ \\
$d\left(s_{i}, p\right)$ & Distance from charger $s_{i}$ to point $p$ \\
$u\left(o_{j}\right)$ & Charging utility of device $o_{j}$ \\
$u\left(s_{i}\right)$ & Charging utility provided by charger $s_{i}$ \\
$e(d), e(p)$ & EMR from distance $d$, EMR at point $p$ \\
$x_{i}$ & Binary indicator denotes whether charger $s_{i}$ is \\
& active or not \\
$R_{t}$ & Hard threshold of EMR safety \\
$\Gamma$ & List of effective active charger sets \\
\hline
\end{tabular}

namely

$$
u\left(o_{j}\right)=c_{1} \sum_{i=1}^{n} P_{r}\left(d\left(s_{i}, o_{j}\right)\right)
$$

where $d\left(s_{i}, o_{j}\right)$ is the distance from the charger $s_{i}$ to the device $o_{j}$, and $c_{1}$ is a predetermined constant.

Similarly, for all rechargeable devices, a charger $s_{i}$ can provide charging utility as

$$
u\left(s_{i}\right)=c_{1} \sum_{j=1}^{m} P_{r}\left(d\left(s_{i}, o_{j}\right)\right) .
$$

For the intensity of EMR, intuitively it is proportional to the received power there. To verify this intuition, we conduct field experiment studies. As shown in Fig. 1, the EMR is nearly proportional to the received power, which can be modeled by $e(d)=c_{2} P_{r}(d)$ where $d$ is the distance and $c_{2}$ is the constant to capture the linear relation. Assuming EMR is also additive, the accumulated EMR at a location $p$ is thus

$$
e(p)=\sum_{s_{i} \in S} e\left(d\left(s_{i}, p\right)\right)=c_{2} \sum_{s_{i} \in S} P_{r}\left(d\left(s_{i}, p\right)\right) .
$$

Some symbols and notations used in this paper are summarized in Table 1.

\section{B. Problem statement}

We start with the following decision problem: given an active charger set $S$, is the EMR safety violated? To answer this question, we have to examine every point on the plane to ensure no place will have the EMR exceeding the hard threshold of EMR safety, which we denote by $R_{t}$. Mathematically, we can express the decision problem as follows

$$
\forall p \in \mathbb{R}^{2}, \quad c_{2} \sum_{s_{i} \in S} P_{r}\left(d\left(s_{i}, p\right)\right) \leq R_{t}
$$

Let $x_{i}$ be a binary indicator that denotes whether charger $s_{i}$ is active or not. For an active charger set specified by $x_{i}$, the above inequality can be rewritten as follows

$$
\forall p \in \mathbb{R}^{2}, \quad c_{2} \sum_{i=1}^{n} P_{r}\left(d\left(s_{i}, p\right)\right) x_{i} \leq R_{t} .
$$

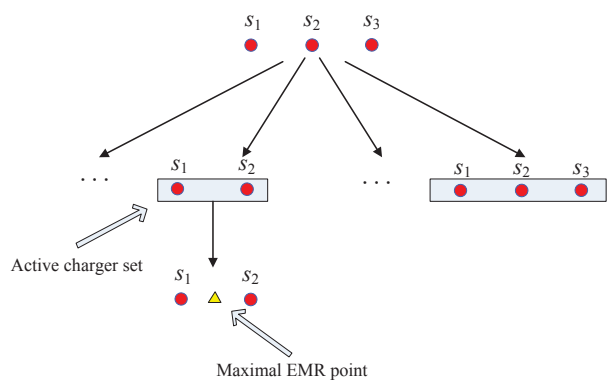

Fig. 2. An illustrative example of constraint conversion. For the set of active chargers $s_{1}$ and $s_{2}$, there will be a maximal EMR point (MEP) located between $s_{1}$ and $s_{2}$, and a corresponding constraint. Different active charger sets will have different MEPs

This inequality actually serves as the constraint of our problem. On the other hand, our optimization goal is to maximize the overall charging utility from all the chargers, namely, $\sum_{i=1}^{n} u\left(s_{i}\right) x_{i}$. The Safe Charging Problem (SCP) can thus be defined as follows

$$
\begin{aligned}
& \text { (SCP) } \quad \max \quad c_{1} \sum_{i=1}^{n}\left(\sum_{j=1}^{m} P_{r}\left(d\left(s_{i}, o_{j}\right)\right)\right) x_{i} \\
& \text { s.t. } \quad \forall p \in \mathbb{R}^{2}, \quad c_{2} \sum_{i=1}^{n} P_{r}\left(d\left(s_{i}, p\right)\right) x_{i} \leq R_{t} \\
& \\
& x_{i} \in\{0,1\} \quad(i=1,2, \ldots, n) .
\end{aligned}
$$

In the above formulation, $x_{i}$ is the optimization variable. We call a location $p \in \mathbb{R}^{2}$ is "safe" if the EMR intensity at this location is below the threshold $R_{t}$, i.e., $c_{2} \sum_{i=1}^{n} P_{r}\left(d\left(s_{i}, p\right)\right) x_{i} \leq R_{t}$, and is "danger" if otherwise.

To solve SCP, we are mainly facing the following challenges. First, the constraint in SCP is imposed on every point on the plane. In other words, there is indeed an infinite number of constraints, which makes the problem extremely difficult. Second, even if we can reduce the number of constraints to a limited number, we will show later that SCP is in the form of a multidimensional 0/1 knapsack problem, which is NP-hard.

Theorem 3.1: SCP is NP-hard.

We omit the proof due to its simplicity and space limitation. In the remainder of this paper, we show how to solve SCP with a near optimal solution.

\section{A NeAr Optimal SOlution}

In this section, we will introduce our solution to SCP and show that the algorithm has near optimal performance. We will first depict the roadmap of our solution, and then present the techniques we applied individually in details.

\section{A. Principles and solution workflow}

As we mentioned before, the major challenge of SCP is the fact that SCP has an infinite number of constraints when optimizing the objective function. To overcome this challenge, we propose two techniques, namely constraint conversion and constraint reduction, to reduce the number of constraints to a limited and tractable number. By this means, SCP will be reduced to a typical multidimensional 0/1 knapsack problem.

The constraint conversion is based on a simple observation. Given a set of active chargers, there will be one point having the maximal EMR intensity, call Maximal EMR Point (MEP) 


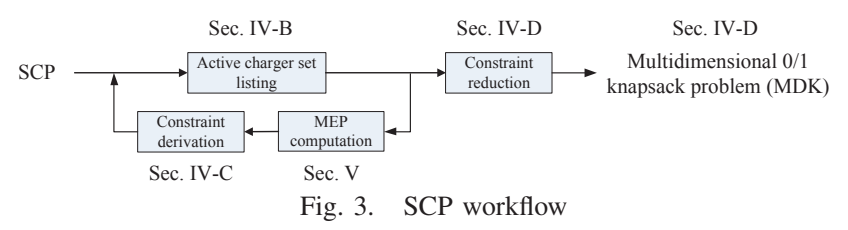

(if there is a set of points with the same maximal EMR intensity, we can arbitrarily pick one of them without affecting the correctness of our solution). When the MEP does not exceed $R_{t}$ and is safe, other locations will be safe too. If the MEP is danger, the constraint is already violated. For example in Fig. 2, suppose there are three chargers $s_{1}, s_{2}$, and $s_{3}$. Consider the active charger set of $s_{1}$ and $s_{2}$ (i.e., $s_{3}$ is inactive). The MEP will be somewhere in between, say location $p$. For this case, we only need to check whether $p$ exceeds $R_{t}$ and derive a corresponding constraint. Note that different active charger sets will have different MEPs, and thus for SCP we shall check all the possible combinations of the active charger sets, compute the MEPs under the active chargers, and rewrite the constraints accordingly. With $n$ chargers, there will be $2^{n}$ MEPs and $2^{n}$ constraints, which is sufficient. Nevertheless, for practical purposes, we find that the number of effective constraints can be significantly reduced, which will be introduced in a later subsection.

Fig. 3 depicts the workflow of our solution. Given an instance of SCP, we first apply the constraint reduction to list all effective active charger sets (Sec. IV-B), compute the MEP under the charger set and derive the corresponding constraint (Sec. IV-C). Then we employ constraint reduction approach to reduce the number of obtained constraints (Sec. IV-D). As such SCP is reformulated to a typical multidimensional 0/1 knapsack problem (MDK), we will give the approximation algorithm for MDK in Sec. IV-D. Since the computation of MEP is quite complex, we skip it in this section and describe it separately in Sec. V.

\section{B. Active charger set listing}

In the active charger set listing, the input is $n$ chargers and their locations, and the output is a list of active charger sets used to derive constraints. Intuitively, each possible charger set will have its MEP and each MEP will lead to a corresponding constraint. In the worst case, for $n$ chargers there will be $2^{n}$ active charger sets and thus $2^{n}$ constraints as well.

This is, however, neither practical nor necessary for further processing. We desire a lightweight list of active charger sets such that the computational overhead is minimized. We will show that the constraints can be reduced to $O\left(n 2^{4 \rho \pi D^{2}}\right)$ where $\rho$ is the charger deployment density, and $D$ is the farthest distance that a charger can reach in Eq. 1, which is much less than $2^{n}$. As shown in Fig. 4, since every MEP must lie in the covered region of a certain charger, we only have to investigate the covered region for each charger. For example, for the disk region covered by $s_{1}$ with radius $D$, an MEP within it can only be charged by chargers with distance less than $D$. In other words, only the chargers with distance to $s_{1}$ less than $2 D$, i.e. $s_{2}$ and $s_{3}$, are able to reach the MEP. The number of these chargers is at most $\rho \pi(2 D)^{2}$. By enumerating

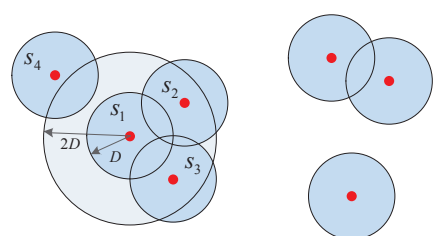

Fig. 4. Illustration of active charger set listing

all possible active charger sets for the covered region of each charger, we obtain in total $O\left(n 2^{4 \rho \pi D^{2}}\right)$ constraints.

\section{Constraint derivation, reduction and MDK}

For each possible active charger set, we can derive the constraint based on its MEP. In the next Sec. V, we will show how to compute an MEP based on a given active charger set. We here use the results directly.

Let $\Gamma$ denote the lightweight list of effective active charger sets we obtained in the last subsection, and $S \in \Gamma$ be an active charger set in $\Gamma$. Suppose the MEP of $S$ is at the location $p$. The constraint associated with $S$ is

$$
c_{2} \sum_{s_{i} \in S} P_{r}\left(d\left(s_{i}, p\right)\right) x_{i} \leq R_{t} .
$$

The obtained constraints by this method can be reduced by removing the following two types of constraints: (i) trivial constraints that can be always satisfied, which means its corresponding active charger set should never lead to an EMR exceeding $R_{t}$; (ii) redundant constraints that can be safely removed if at least one of the subsets of its corresponding active charger set leads to an EMR exceeding $R_{t}$. For example, if active charger set of $s_{1}$ and $s_{2}$ has already violated EMR safety, it is needless to include the constraint of its superset of $s_{1}, s_{2}$ and $s_{3}$.

With the reduced constraints set, which we still denote by $\Gamma$ for convenience, $\mathrm{SCP}$ is reformulated as

$$
\begin{array}{ccc}
\text { (SCP) } \quad \max \quad c_{1} \sum_{i=1}^{n}\left(\sum_{j=1}^{m} P_{r}\left(d\left(s_{i}, o_{j}\right)\right)\right) x_{i} \\
\text { s.t. } \quad \forall S \in \Gamma, \quad c_{2} \sum_{s_{i} \in S} P_{r}\left(d\left(s_{i}, p\right)\right) x_{i} \leq R_{t} \\
& x_{i} \in\{0,1\} \quad(i=1,2, \ldots, n) .
\end{array}
$$

This is a typical MDK problem [25]. When the number of constraint $|\Gamma| \geq 2$, there does not exist a FPTAS unless $P=N P$ [25]. We here apply an algorithm proposed in [33] to obtain an approximation solution.

\section{Algorithm description and results}

In this section, we introduce our approximation algorithm using pseudo-code and present the analytical results for the algorithm, demonstrating its near optimal performance.

Algorithm 1 presents the algorithm pseudo-code. The input of our algorithm is the set of chargers, devices and their locations. The output of the algorithm is the binary indicator $x_{i}$ to control whether a charger should be active or not. The following theorem describes the performance of Algorithm 1.

Theorem 4.1: The time complexity of Algorithm 1 is $\left.O\left(n^{4}\left(\epsilon^{-2}+\epsilon^{-3 / 2} n\right)+n\left(\frac{n}{\epsilon}\right)^{|\Gamma|}\right)\right)$. The output of Algorithm 1 is a feasible solution of SCP, and outperforms the optimal solution to SCP with a smaller EMR threshold $(1-\epsilon) R_{t}$.

Proof: Its complete proof is available in [34]. 


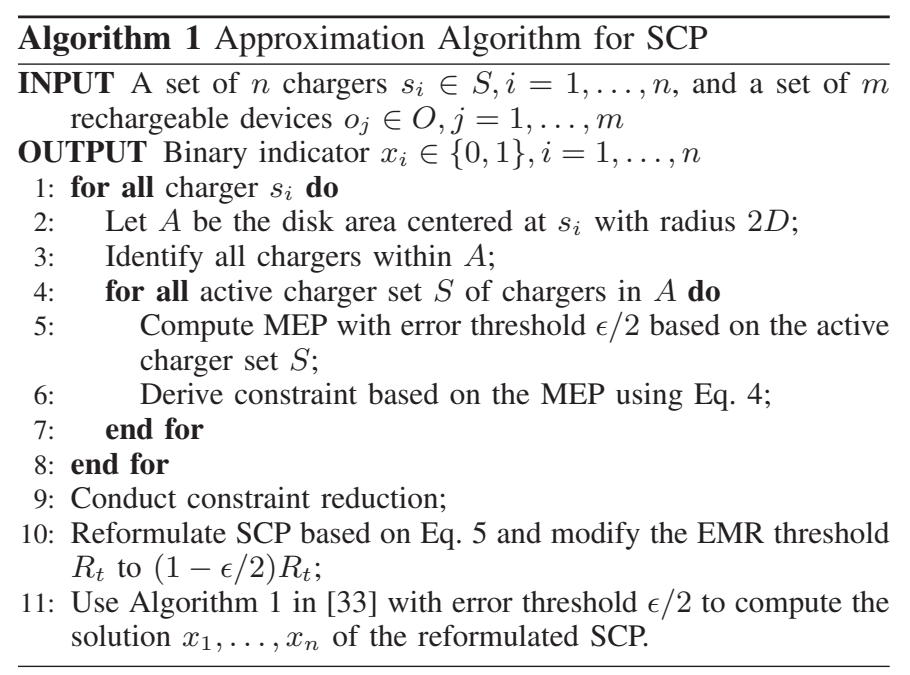

\section{MEP COMPUTATION}

In this section, we compute MEP for a given active charger set. This is a major but difficult problem when solving SCP. The challenges are mainly due to the fact that the objective function $e(p)$ in Eq. 2 is non-convex, and thus there is no standard solution to be global optimal. In addition, the search space of MEP is continuous, but the output is a single point. Appropriate discretization method is needed to strike the tradeoff between the computation precision and overhead.

In the remainder of this section, we first give the main results, and present our approximation algorithm in details.

\section{A. Main results}

Our results can be summarized as follows.

Theorem 5.1: For any given small number $\epsilon$, the solution $p^{\#}$ obtained by our algorithm and the optimal solution $p^{*}$ satisfies

$$
(1-\epsilon) e\left(p^{*}\right) \leq e\left(p^{\#}\right) \leq e\left(p^{*}\right) .
$$

The computational time complexity is $O\left(\epsilon^{-2} n^{3}+\epsilon^{-3 / 2} n^{4}\right)$, and the space requirement is $O\left(\epsilon^{-1 / 2} n \log n\right)$ where $n$ is the number of chargers in the input active charger set.

This shows that the EMR of our solution point $p^{\#}$ can be arbitrarily close to that of the optimal one, and our algorithm is thus a fully polynomial time approximation scheme (FPTAS).

\section{B. Algorithm overview}

The key issue in MEP computation is to approximate the non-convex EMR function $e(p)$ by convex ones so that the problem is transferred into a traditional Fermat-Weber problem [26]. During the approximation, we should guarantee each step only introduces a bounded error such that the overall algorithm performance can be bounded as well.

Fig. 5 depicts the workflow of our algorithm. We first use a piecewise linear function $\varepsilon(d)$ to approximate $e(d)$ (Sec. VC). By this means, the covered area of a charger is partitioned to many subareas. Subareas of different chargers will overlap to further partition the area, and each partitioned subarea has a convex objective function (Sec. V-D). Nevertheless, some subareas may become a non-convex shape, and we thus expand

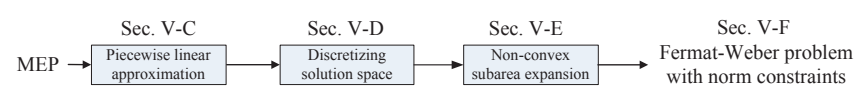

Fig. 5. MEP workflow

the subareas to convex ones in the third step (Sec. V-E). By these means, the MEP computation problem can be transferred into a traditional Fermat-Weber problem with norm constraints (Sec. V-F). The MEP of the whole area is then among these subarea MEPs and easy to find.

As the goal of each procedure is to convert MEP to the Fermat-Weber problem with norm constraints, we present the formal definition of this problem in advance as follows, in order for a better understanding of these procedures.

Definition 5.1: Fermat-Weber problem with Norm Constraints $(\boldsymbol{F W N C})$ : Let $S=\left\{s_{1}, s_{2}, \ldots, s_{n}\right\}$ be a set of points in $\mathbb{R}^{2}$, the Weber-Fermat problem is a facility problem that aims to find the point $p$ such that

$$
\begin{aligned}
& \min \sum_{i \in n} w_{i} d\left(s_{i}, p\right) \\
& \text { s.t. } \quad d\left(s_{i}, p\right) \leq C_{i}, i=1,2, \ldots, n .
\end{aligned}
$$

where $w_{i}$ and $C_{i}$ are constants.

In the remaining subsections of this paper, we will present the detailed design of these procedures.

\section{Piecewise linear approximation of $e(d)$}

Essentially, we use multiple piecewise linear segments $\varepsilon(d)$ to approximate the EMR function $e(d)$ (recall $e(d)=$ $\left.c_{2} P_{r}(d)\right)$, trying to bound the approximation error $\varepsilon(d)-e(d)$ and the computational overhead.

The basic idea of the approximation $\varepsilon(d)$ is illustrated in Fig. 6. Let the vector $L=\left\{\ell_{0}, \ell_{1}, \ldots, \ell_{K}\right\}$ be the end points of $K$ linear segments in an increasing sequence. The parameter $K$ is the number of segments that controls the approximation error. In the example of Fig. $6, K$ is equal to 3 . And in general speaking, a larger $K$ will result in a smaller approximation error but introduces more computation overhead.

Definition 5.2: Setting $\ell_{0}=0$ and $\ell_{K}=D$, the piecewise approximation function $\varepsilon(d)$ can be defined as

$$
\varepsilon(d)= \begin{cases}-w_{k} d+\phi_{k}, & \ell_{k-1} \leq d \leq \ell_{k}(k=1, \ldots, K) \\ 0, & d>D\end{cases}
$$

where $\phi_{k}=\frac{e\left(\ell_{k}\right) \ell_{k+1}-e\left(\ell_{k+1}\right) \ell_{k}}{\ell_{k+1}-\ell_{k}}\left(\phi_{k}>0\right)$ and $w_{k}=$ $-\frac{e\left(\ell_{k+1}\right)-e\left(\ell_{k}\right)}{\ell_{k+1}-\ell_{k}}\left(w_{k}>0\right)$ when $k \leq K$, and otherwise
$\phi_{k}=w_{k}=0$.

Definition 5.3: To bound the approximation error, we set $\ell_{k+1}$ (if the obtained $\ell_{k+1} \geq D$, set $K=k+1, \ell_{k+1}=D$ ) sequentially based on $\ell_{k}\left(\ell_{0}=0\right)$ in Eq. 7 as

$\ell_{k+1}=\frac{1}{4}\left(3 x_{0}-2 \ell_{k}+\beta+\sqrt{\left(3 x_{0}-2 \ell_{k}+\beta\right)\left(3 x_{0}+6 \ell_{k}+9 \beta\right)}\right)-\beta$

where $\beta$ is a constant in Eq. 1, and $x_{0}$ is one of the roots to the following cubic equation

$$
\frac{1-\epsilon}{\left(\ell_{k}+\beta\right)^{2}}\left(x_{0}+\beta\right)^{3}-3 x_{0}+2 \ell_{k}-\beta=0
$$

that satisfies $x_{0}>\ell_{k}$. 


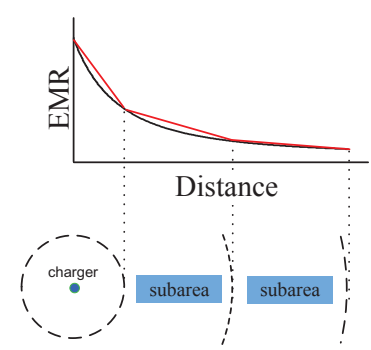

Fig. 6. Illustration of piecewise linear approximation

By these definitions, we have

Theorem 5.2: Setting $\ell_{k}$ by Eq. 8, we have the approximation errors as

$$
e(d) \leq \varepsilon(d) \leq \frac{e(d)}{1-\epsilon} .
$$

Theorem 5.3: If $\epsilon \rightarrow 0$, the number of linear segments $K$ is subject to

$$
\frac{\sqrt{3}}{4}\left(1-\frac{1}{(1+D / \beta)^{2}}\right) \epsilon^{-1 / 2}<K<\frac{\sqrt{3}}{4}\left((1+D / \beta)^{2}-1\right) \epsilon^{-1 / 2} .
$$

In other words, we have $K=\Theta\left(\epsilon^{-1 / 2}\right)$.

\section{Discretize solution space}

In this part, we show how to discretize and confine the search space for MEP. The goal is to show that with the approximated EMR function $\varepsilon(d)$, the MEP is reformulated as an optimization problem with convex objective function.

By $\varepsilon(d)$, the covered area of a charger $s$, denoted as $A_{s}$, is partitioned to $K$ concentric sub-areas denoted as $A_{s}(k), k=$ $1, \ldots, K$. Obviously given the active charger set $S$, there will be at most $K|S|$ concentric subareas which may overlap with each other. By computational geometry [35], $|S|$ chargers will partition the whole plane to at most $Z$ subarea faces where

$$
Z \leq(K|S|)^{2}-K|S|+2 \leq K^{2}|S|^{2} .
$$

An illustration of such subarea faces with three chargers is depicted in Fig. 7. Note that we do not consider the area not covered by any chargers as obviously there has no EMR and MEP cannot be there.

By such partition, MEP for a given active charger set becomes to find MEP from each subarea face, and among these MEPs find the one with the largest EMR. As the second step is straightforward, we here focus on the first step. Denote a subarea face overlapped by several chargers as $\mathcal{F}(\kappa)=\bigcap_{s_{i} \in S} A_{s_{i}}\left(k_{i}\right)$ where $\kappa=\left(k_{1}, k_{2}, \ldots k_{|S|}\right)$ is a vector indicating the index of concentric subarea that shape the face. In Fig.7, the face is shaped by $s_{1}$ 's second subarea, $s_{2}$ 's second subarea, and $s_{3}$ 's third subarea, and thus it can be expressed as $\mathcal{F}(\kappa)=A_{s_{1}}(2) \cap A_{s_{2}}(2) \cap A_{s_{3}}(3)$ where $\kappa=\{2,2,3\}$. The accumulated EMR approximation for a location $p$ in $\mathcal{F}(\kappa)$ is

$$
\varepsilon(p)=\sum_{s_{i} \in S}\left(-w_{k_{i}} d\left(s_{i}, p\right)+\phi_{k_{i}}\right), p \in \mathcal{F}(\kappa) .
$$

Note $w_{k_{i}}$ and $\phi_{k_{i}}$ defined in Eq. 7 are both constants within the concentric subarea $A_{s_{i}}\left(k_{i}\right)$. And therefore maximizing $\varepsilon(p)$ is equivalent to minimizing $\sum_{s_{i} \in S} w_{k_{i}} d\left(s_{i}, p\right)$. In other words, MEP of an active charger set can be reformulated as

$$
\max _{\forall \mathcal{F}(\kappa)}\left\{\varepsilon(p) \mid p=\min _{p \in \mathcal{F}(\kappa)} \sum_{s_{i} \in S} w_{k_{i}} d\left(s_{i}, p\right)\right\} .
$$

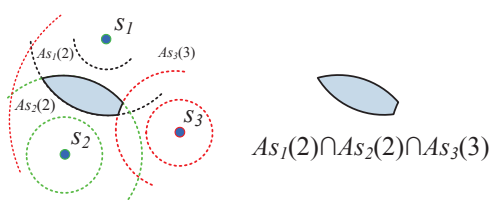

Fig. 7. Illustration of discretized charging area

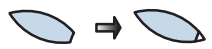

Fig. 8. Illustration of area expansion

\section{E. Area expansion}

In the last subsection, we can find that MEP is reformulated with a convex objective function $\sum_{s_{i} \in S} w_{k_{i}} d\left(s_{i}, p\right)$, which allows optimization method to apply. However, by partitions in the last subsection the subarea face $\bigcap_{s_{i} \in S} A_{s_{i}}\left(k_{i}\right)$ may become non-convex (See the example in Fig. 7), which will be dealt with in this subsection.

Definition 5.4: Denoting by $\Omega_{s_{i}}\left(k_{i}\right)$ the minimal enclosing disk of $A_{s_{i}}\left(k_{i}\right), \Omega_{s_{i}}\left(k_{i}\right)$ is indeed the union of all concentric subareas no more than $k_{i}$, i.e., $\Omega_{s_{i}}\left(k_{i}\right)=\bigcup_{k<k_{i}} A_{s_{i}}(k)$. The expanded area $\Lambda(\kappa)$ for a subarea face $\mathcal{F}(\kappa)$ is defined as

$$
\Lambda(\kappa)=\bigcap_{s_{i} \in S} \Omega_{s_{i}}\left(k_{i}\right)=\bigcap_{s_{i} \in S}\left(\bigcup_{k \leq k_{i}} A_{s_{i}}(k)\right)
$$

where $\kappa=\left\{k_{1}, \ldots, k_{|S|}\right\}$.

Fig. 8 illustrates an example of area expansion based on the example in Fig. 7.

Though $\mathcal{F}(\kappa)$ is expanded to $\Lambda(\kappa)$, the solution to MEP will not change, as the following theorem indicates.

Theorem 5.4: Suppose $p^{*}$ and $\bar{p}^{*}$ are optimal solutions to the reformulated MEP defined in Eq. 10 before and after area expansion for each subarea, then $\varepsilon\left(p^{*}\right)=\varepsilon\left(\bar{p}^{*}\right)$.

Proof: Its complete proof is available in [34].

We emphasize that Theorem 5.4 is not an immediate observation, but a crucial conclusion based on the convexity and monotonic decreasing properties of the EMR function as well as the properties of our piecewise linear approximation.

We therefore further reformulate MEP in each subarea as

$$
\begin{aligned}
& \min \sum_{s_{i} \in S} w_{k_{i}} d\left(s_{i}, p\right) \\
& \text { s.t. } \quad d\left(s_{i}, p\right) \leq \ell_{k_{i}}, i=1,2, \ldots,|S| .
\end{aligned}
$$

Notice that the above constraints stem from the definition of expanded area $\Lambda(\kappa)$. In the next subsection, we will show how to solve it by a modified Fermat-Weber problem algorithm.

\section{F. Fermat-Weber problem with norm constraints}

Comparing our MEP in Eq. 12 and the Fermat-Weber problem with norm constraints (FWNC) in Eq. 6 we find that they are exactly the same. FWNC is a traditional problem that has been widely studied [26]. There is, however, no standard approximation solution for the problem. Therefore, we tailor the approximation algorithm to unweighted FermatWeber problem constrained to a polyhedron in [36] to our case. The main difference between them lies in the boundary search. The algorithm in [36] simply uses a binary search to 

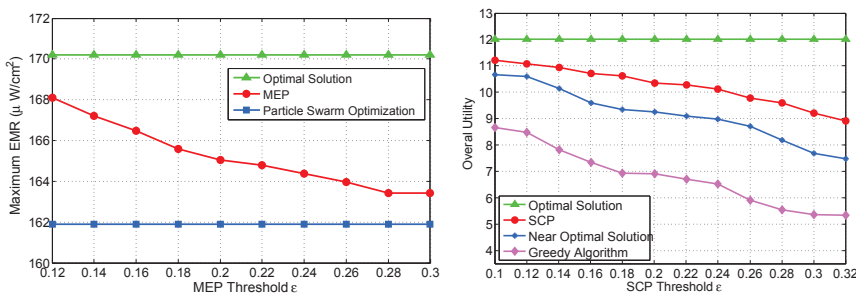

Fig. 9. Maximal EMR vs. MEP Fig. 10. Threshold $\epsilon$ Threshold $\epsilon$

Overall Utility vs. SCP

find the optimal solution on the line segments of boundaries. In contrast, our algorithm searches all the boundary arcs and adopts Lagrange multiplier method to optimize the objective function. We refer readers to [34] for details.

For our approximation algorithm, we have

Theorem 5.5: For any given small number $\epsilon$, our algorithm to FWNC can achieve $(1-\epsilon)$ approximation ratio in deterministic $O\left(\epsilon^{-1} n+\epsilon^{-1 / 2} n^{2}\right)$ time and $O\left(\epsilon^{-1 / 2} n \log n\right)$ space.

\section{NUMERICAL RESULTS}

In this section, we first conduct extensive simulations to evaluate our proposed algorithms under different parameter settings, and then reveal insights of the algorithm performance.

\section{A. Evaluation setup}

We assume that there are 12 chargers uniformly deployed over a $100 m * 100 m$ 2D square area and 100 devices randomly deployed in the area too. We set $\alpha=100, \beta=40$ and $D=60$ for the charging model, and $c_{1}=1$ for the EMR model. For the utility model, we simply set $c_{2}=0.001$. Moreover, the error threshold of the SCP algorithm is $\epsilon=0.12$, and the EMR threshold is $R_{t}=150$. Note that the result is averaged by 100 instances with different random seeds and device deployments.

\section{B. Baseline setup}

In Sec. VI-C1, we compare the MEP algorithm to the particle swarm optimization (PSO) with the number of particles set to be 20 and the loop count be 200 . In addition, we use a fine-grained exhaustive search method to find the MEP on the plane, and take its output as the optimal solution.

In Sec. VI-C2, VI-C3, VI-C4 and VI-D, we compare the SCP solution to three different algorithms. The first is the optimal solution obtained by enumerating all possible activations of chargers in SCP. Note that here the constraints in SCP are outputs of the optimal solution for MEP used in Sec. VI-C1. The second is the near optimal solution. It is identical to the optimal solution except that its EMR threshold is set to be $(1-\epsilon) R_{t}$. The third is a newly designed greedy algorithm for SCP whose constraints are derived based on our approximation algorithm for MEP. Particularly, the greedy algorithm turns on the charger that yields the maximum overall additional utility for all devices while does not violate the EMR safety requirement at each step. Such process repeats until no further activation is possible in terms of the EMR safety.
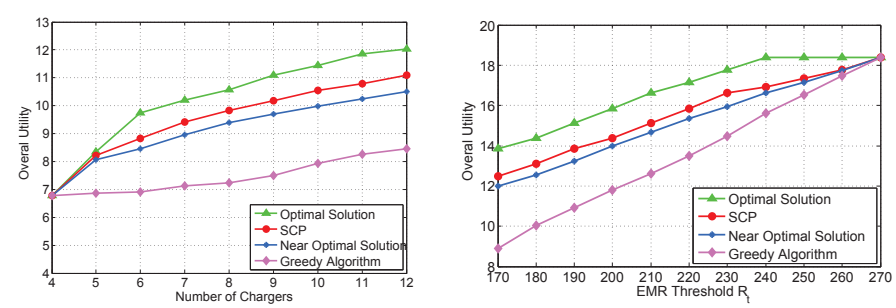

Fig. 11. Overall Utility vs. Charger Fig. 12. Overall Utility vs. EMR Number

\section{Performance comparison}

In this subsection, we examine the performance of our approximation algorithm for MEP in terms of approximation threshold $\epsilon$, and that for SCP under various designs with different system parameters including the threshold $\epsilon$, the charger number and the EMR threshold $R_{t}$.

1) Impact of the MEP threshold $\epsilon$ : To show the efficiency of our MEP algorithm, we consider 50 chargers and 100 devices uniformly deployed over a $500 m * 500 m$ 2D square area, and investigate the performance of our approximation algorithm for MEP and other two baseline algorithms in terms of the MEP threshold $\epsilon$. As illustrated in Fig. 9, the outputs of the optimal solution and the PSO are 170.2 and 161.9 respectively and remain constant. The maximal EMR computed by our algorithm decreases with an increasing $\epsilon$. It is always greater than that of the PSO and is at most $4 \%$ smaller than that of the optimal solution for $0.12 \leq \epsilon \leq 0.3$. This indicates that the approximation bound $(1-\epsilon)$ strictly holds.

2) Impact of the SCP threshold $\epsilon$ : We proceed to examine the influence of the SCP threshold $\epsilon$ on the overall utility and plot the results in Fig. 10. As can be seen, the overall utility of the optimal solution is constant and equal to 12. Our SCP algorithm always outperforms that of the near optimal solution, and the performance gap with the optimal solution diminishes when $\epsilon$ decreases and is equal to $6.7 \%$ when $\epsilon=0.1$. This observation validates our theoretical findings. In addition, the greedy algorithm has the worst performance, which is roughly $34.6 \%$ below that of our SCP algorithm on average.

3) Impact of charger number: We are also interested in the impact of the charger number on overall utility. Fig. 11 shows that all algorithms have the same performance when the charger number is 4 . This is because the activation of all 4 chargers will never damage the EMR safety and thus there is no room for improvement. Besides, the overall utility of every algorithm increases smoothly with the number of chargers, and our SCP algorithm performs better than the near optimal algorithm, and the performance difference from the optimal one is at most $9.3 \%$. The gap between the SCP algorithm and the greedy algorithm can be as large as $26.4 \%$.

4) Impact of EMR threshold $R_{t}$ : We study the effect of the EMR threshold $R_{t}$ to the overall utility in this subsection. As shown in Fig. 12, not surprisingly, the overall utility of all considered solutions grows with an increasing $R_{t}$. The performance of our SCP algorithm also outperforms the near optimal solution. Overall, the optimal solution is nearly $7.7 \%$ higher than that of our SCP algorithm, which in turn enjoys an average performance gain of $14.9 \%$ over the greedy algorithm. 

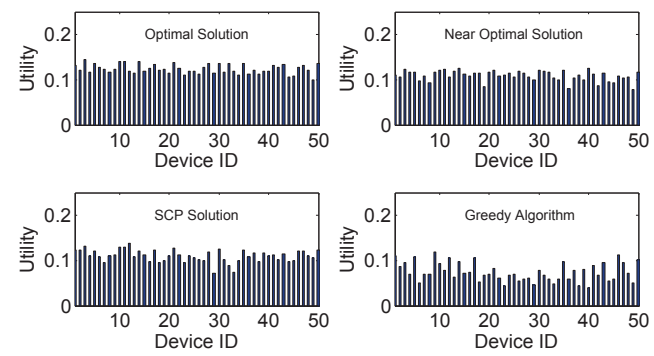

Fig. 13. Utility of devices for four solutions

Furthermore, when $R_{t}$ exceeds 240, the activation of all 12 chargers is allowed for the sake of EMR safety, and thereby the overall utility of the optimal solution reaches the maximum 18.4 and remains constant from then on. The same situation occurs to the other three algorithms when $R_{t}$ exceeds 270 .

\section{Insights}

In this section, we explain why our proposed scheme can obtain a high overall utility gain. We conduct a simulation and observe the utility of each of 50 devices, as shown in Fig. 13. Since the charging utility of a device is proportional to the EMR there (please refer to their definitions in Sec. III-A), the utility distribution of devices actually reflects the EMR distribution for the locations of devices. Intuitively, if all the EMRs at the locations of devices are quite close to the EMR threshold $R_{t}$, which means that the EMRs are balanced among these locations, the overall utility will be high. From the top two sub-figures of Fig. 13, we can see that the utility of all 50 devices are nearly uniform, and close to 150 and 132 respectively, which are the theoretical maximum utility constrained by their EMR safety requirements. As for the bottom two sub-figures, the greedy algorithm has utility distribution with a higher variance. This is because the greedy algorithm is conducted in such a way that it totally overlooks the balance of EMR distribution. The EMRs at certain points on the plane shall rise much quickly than others during the greedy process of activating new chargers, and soon approach $R_{t}$ and disable further charger activation. Hence, its overall achieved utility is low. Conversely, our SCP solution performs in a reasonable manner so that the utility is balanced among devices and thus is improved significantly.

\section{FIELD EXPERIMENTS}

In this section, we conduct field experiments to validate our theoretical results.

\section{A. Experimental testbed}

Fig. 14(a) shows the indoor experimental testbed. We utilize 8 chargers (TX91501 transmitters produced by Powercast [3]) which are deployed on the vertices and middle points of edges of a $2.4 m * 2.4 m$ square area. In addition, we place one wireless rechargeable sensor node (see Fig. 14(b)) at the center of the square area, and the other to the right side of the first one with distance $0.4 \mathrm{~m}$. We use an RF field strength meter (see Fig. 14(c)) to measure the intensity of EMR.

It is noteworthy that all the chargers are actually directional. With reasonable precision, we model their charging region as a sector with angle $60^{\circ}$ and radius 4 . The orientation of chargers should thus greatly impact the EMR distribution of the space. Suppose the chargers are numbered from top to bottom and from left to right as shown in Fig. 14(a). We rotate chargers from 1 to 8 such that the angles between their orientation and the positive horizontal line are $296.56^{\circ}, 296.56^{\circ}, 243.44^{\circ}$, $26.56^{\circ}, 153.44^{\circ}, 63.44^{\circ}, 116.56^{\circ}$ and $116.56^{\circ}$, respectively, in order to enhance the charging efficiency in the square area.

The computer controls the power supply through a power manager. The sensor nodes record their received power and send the information to an access point (AP) connecting to the computer. The AP then reports the data to the computer for analysis and decision.

\section{B. Adapted algorithm description}

We make the following adaptations to our SCP algorithm considering real situations. First, we adjust our SCP algorithm to the case under directional chargers. This can be done by modifying our MEP algorithm. We omit the details to save space. Second, to alleviate the error incurred by modeling of directional chargers, environmental variation, etc., we let the two sensors sample the charging power from each charger at the beginning of the algorithm. Then we perform our SCP algorithm based on the sampled values.

\section{Experimental results}

As Fig. 15 illustrates, we compare the computed utility based on sampling with real utility under three different values of $R_{t}$. Note that in this figure, Node 1 refers to the node located at the center of the area and Node 2 refers to the other. We can see that the computed utility of both nodes is always larger than the real utility, but the difference between them is quite small and no more than $7.5 \%$. This observation supports the power additivity assumption and the effectiveness of our sampling approach. Furthermore, the gap between these two solutions tends to increase when the utility grows. This is likely due to the charging property of the capacitors in sensors.

Suppose the EMR threshold $R_{t}$ is $125 \mu \mathrm{W} / \mathrm{cm}^{2}$, we turn on charger 2, 4, 5, 6, 7 and 8 according to the output of our adapted algorithm. Then we measure the EMR values at $9 * 9$ grid points of the square region, and plot them in Fig. 16 to visualize the EMR distribution of the area in an approximation manner. We observe that the EMR peaks at the location of charger 5 and is equal to $116.7 \mu \mathrm{W} / \mathrm{cm}^{2}$ and thus less than $R_{t}$. This fact confirms the correctness of our SCP algorithm.

\section{RELATED WORK}

In this section, we briefly review works regarding scenarios with stationary chargers. Recently, Intel developed the wireless identification and sensing platform (WISP) by integrating RFID tags with sensing and computing components. The RFID tags can be wirelessly charged by readers. Buettner et al. explored this technology to recognize human activities in [9], and highlighted applications such as elderly care in [10]. In [11], Powercast developed a wireless power platform to work with wireless sensor network. Their objective was to help 


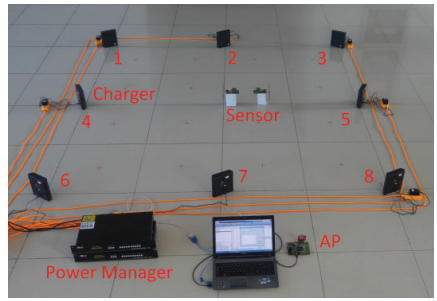

(a)

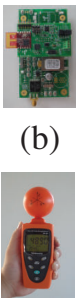

(c)

Fig. 14. Illustration of field experiment

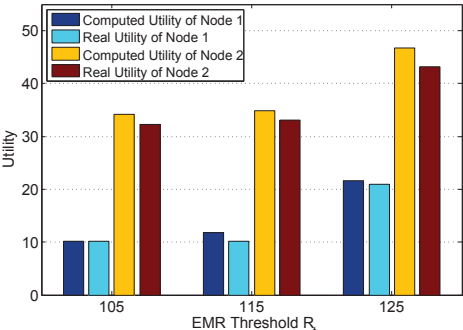

Fig. 15. Utility vs. EMR Threshold $R_{t}$

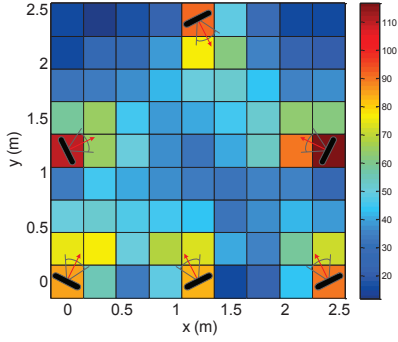

Fig. 16. An example of EMR distribution monitor temperature and humidity at a zoo without disrupting the animal exhibit. This company also offered a solution for data center environmental monitoring by constructing a network of chargers [12]. He et al. [13] studied the energy provisioning problem, i.e., how to deploy chargers to provide sufficient energy to static/mobile devices in wireless rechargeable sensor networks. In [14], Wicaksono et al. considered the power interference when allocating frequency bands to adjacent stationary chargers. All the above works overlooked EMR safety.

\section{CONCLUSION}

In this paper, we have studied the problem of maximizing the charging utility under the constraints of EMR safety. Considering the complexity of the original problem, we broke it down into two related subproblems, i.e., SCP and MEP. For MEP, we proposed a series of novel techniques to transform it into a classical problem FWNC. These techniques combined with our tailored approximation algorithm for FWNC form a $(1-\epsilon)$ solution. Based on the powerful results of MEP, we presented a near optimal solution to SCP. To evaluate the effectiveness of our solution, we conducted both extensive simulations and field experiments. All of their results corroborated our analytical findings. This solution could be incorporated into many systems to harness the detrimental impact of EMR.

\section{ACKNOWLEDGMENT}

This work is supported by National 973 project of China under Grants No.2012CB316200 and No.2014CB340300, National Natural Science Foundation of China under Grants No.61133006, No.61373130, No.61321491, No.61170247 and No.613230428, National Key Project of China under Grant No.2013ZX01033002-003, Research and Innovation Project for College Graduate Students of Jiangsu Province in 2012 under Grant No.CXZZ12_0056, and the Shanghai Recruitment Program of Global Experts.

\section{REFERENCES}

[1] A. Kurs et al., "Wireless power transfer via strongly coupled magnetic resonances," science, vol. 317, no. 5834, pp. 83-86, 2007.

[2] "http://www.seattle.intel-research.net/wisp/."

[3] "www.powercastco.com."

[4] "www.powermat.com."

[5] "http://www.laptopmag.com/reviews/laptops/dell-latitude-3330.aspx."

[6] "http://evworld.com/news.cfm?newsid=24420."

[7] "http://www.friendsofcrc.ca/projects/sharp/sharp.html."

[8] "http://equity.co.kr/upfile/issue/2012/05/10/1336611859340.pdf."

[9] M. Buettner et al., "Recognizing daily activities with RFID-based sensors," in Ubicomp, 2009.
[10] M. Buettner et al., "Revisiting smart dust with RFID sensor networks," in HotNets-VII, 2008.

[11] C. Greene et al., "Making wireless sensor networks truly wireless using RF power."

[12] "http://www.powercastsensors.com/category/applications/page/2/."

[13] S. He et al., "Energy provisioning in wireless rechargeable sensor networks," in INFOCOM, 2011, pp. 2006-2014.

[14] R. P. Wicaksono et al., "Wireless Grid: enabling ubiquitous sensor networks with wireless energy supply," pp. 1-5, 2011.

[15] "http://powercastco.com/pdf/2009sensorsexpo2.pdf."

[16] J. G. Shearer et al., "Power transmission network," Nov. 30 2010, uS Patent 7,844,306.

[17] H. Dai et al., "Using minimum mobile chargers to keep large-scale wireless rechargeable sensor networks running forever," in ICCCN, 2013.

[18] H. Dai et al., "Impact of mobility on energy provisioning in wireless rechargeable sensor networks," in WCNC, 2013.

[19] H. Dai et al., "Near optimal charging and scheduling scheme for stochastic event capture with rechargeable sensors," in MASS, 2013.

[20] H. Dai et al., "Practical scheduling for stochastic event capture in wireless rechargeable sensor networks," in WCNC, 2013.

[21] A. Ahlbom et al., "Guidelines for limiting exposure to time-varying electric, magnetic, and electromagnetic fields (up to $300 \mathrm{GHz}$ ). international commission on non-ionizing radiation protection." Health Phys, vol. 74, no. 4, pp. 494-522, 1998.

[22] M. OLTEANU et al., "Dangerous temperature increase from EM radiation around metallic implants," 2012.

[23] M. Havas et al., "Provocation study using heart rate variability shows microwave radiation from $2.4 \mathrm{GHz}$ cordless phone affects autonomic nervous system," European Journal of Oncology Library, vol. 5, 2010.

[24] M. P. Ntzouni et al., "Transient and cumulative memory impairments induced by GSM $1.8 \mathrm{GHz}$ cell phone signal in a mouse model," Electromagnetic biology and medicine, 2013.

[25] A. Fréville, "The multidimensional 0-1 knapsack problem: An overview," European Journal of Operational Research, 2004.

[26] A. P. Hurter et al., "Solutions of constrained location problems," Management Science, vol. 22, no. 1, pp. 51-56, 1975.

[27] Y. P. Shkolnikov et al., "Electromagnetic interference and exposure from household wireless networks," in PSES. IEEE, 2011, pp. 1-5.

[28] O. P. Gandhi et al., "Exposure limits: The underestimation of absorbed cell phone radiation, especially in children," Electromagnetic Biology and Medicine, vol. 31, no. 1, pp. 34-51, 2012.

[29] M. Edwards et al., "Effects of heat on embryos and foetuses." International journal of hyperthermia: the official journal of European Society for Hyperthermic Oncology, North American Hyperthermia Group, vol. 19, no. 3, pp. 295-324, 2002.

[30] A. Karinen et al., "Mobile phone radiation might alter protein expression in human skin," BMC genomics, vol. 9, no. 1, p. 77, 2008.

[31] H. Nittby et al., "Exposure to radiation from global system for mobile communications at $1,800 \mathrm{MHz}$ significantly changes gene expression in rat hippocampus and cortex," The Environmentalist, vol. 28, no. 4, pp. 458-465, 2008.

[32] "http://www.moh.gov.cn/zwgkzt/pgw/201212/34317.shtml."

[33] W. Ge et al., "MIMO-Pipe modeling and scheduling for efficient interference management in multihop mimo networks," TOVT, 2010.

[34] "http://gps.nju.edu.cn/hpdai/dh/scp-tr.pdf."

[35] M. De Berg et al., Computational geometry: algorithms and applications. Springer, 2008.

[36] P. Bose et al., "Fast approximations for sums of distances, clustering and the fermat-weber problem," Computational Geometry, 2003. 